Schmerz $2021 \cdot 35: 80-82$

https://doi.org/10.1007/s00482-021-00542-9

Angenommen: 11. Februar 2021

๑) Springer Medizin Verlag GmbH, ein Teil von Springer Nature 2021

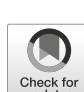

\section{B. Zernikow ${ }^{1,2}$}

' Deutsches Kinderschmerzzentrum, Vestische Kinder- und Jugendklinik Datteln, Universität Witten/Herdecke, Datteln, Deutschland

${ }^{2}$ Lehrstuhl für Kinderschmerztherapie und Pädiatrische Palliativmedizin, Universität Witten/Herdecke, Witten, Deutschland

\title{
Chronische Schmerzen bei Kindern und Jugendlichen
}

\section{Die stille Epidemie}

Psychosomatische Erkrankungen wie Adipositas und chronische Schmerzen betreffen immer mehr Kinder und Jugendliche in den entwickelten Industrieländern wie Deutschland. Zwar rücken oft andere Erkrankung, wie z. B. Krebserkrankungen oder komplexe angeborene Herzfehler in den Fokus, weil eine sehr wirksame Öffentlichkeitsarbeit umgesetzt wird (https://www.kinderherzen. de/herzplakat-bundesweite-kampagnestartet/), doch diese Erkrankungen haben im Kindesalter seit Jahrzehnten eine stabile Prävalenz; Kinder mit onkologischen Erkrankungen oder komplexen Herzfehlern werden in exzellenten Versorgungsnetzwerken sowie ausgewiesenen Fachzentren versorgt. Chronische Schmerzen bei Kindern und Jugendlichen hingegen zeigen einen deutlichen Prävalenzanstieg, die spezialisierte Versorgung ist mangelhaft [1]. Dennoch bleibt diese „stille“ Epidemie weitgehend unbemerkt von der Öffentlichkeit.

Kinder und Jugendliche leiden im Verborgenen. Sie fehlen wegen der chronischen Schmerzen oft in der Schule, schöpfen so ihr Bildungspotenzial nicht aus. Sie entwickeln psychische Komorbiditäten wie Depression und Ängste, oder vorbestehende psychische Erkrankungen werden durch die chronischen Schmerzen verstärkt. Schmerzbedingt erfahren sie eine massive Einschränkung ihrer Alltagsaktivitäten. Ihre Eltern können ihrer Arbeit nur noch unregelmäßig nachgehen, weil sie immer wieder Fehltage aufweisen, wenn sie sich zu Hause um ihr chronisch schmerzkrankes Kind kümmern. Und, last but not least, sind chronische Schmerzen im Kindesalter ein Risikofaktor für chronische Schmerzen im Erwachsenenalter.

Nehmen Kinder mit chronischen Schmerzen das Gesundheitssystem in Anspruch, erhalten sie nicht selten zu viele, $\mathrm{zu}$ risikoreiche und die falschen diagnostischen Untersuchungen oder therapeutischen Maßnahmen [3]. Andererseits stehen spezialisierte Einrichtungen zur Diagnostik und Therapie chronischer Schmerzen im Kindes- und Jugendalter nicht flächendeckend zur Verfügung [1].

Was ist also zu tun? Was benötigen chronisch schmerzkranke Kinder und Jugendliche sowie deren Familien?

1. Schon in Kindergarten und Schule sollten präventive Ansätze die Regel sein. Wichtige Elemente könnten sein: Selbstfürsorge, Spaß an Bewegung, Resilienzsteigerung, Umgang mit Stress und neuen Medien, Gefahren der Selbstmedikation sowie die Neurobiologie des Schmerzes.

2. In Deutschland ist die Primärversorgung bei den niedergelassenen Kinder- und Jugendärzt ${ }^{*}$ innen anders als in anderen europäischen Ländern sehr gut geregelt und auf einem sehr hohen Niveau. Leider haben die niedergelassenen Kolleginnen oft nicht die Zeit, eine ausführliche psychosomatische Evaluation durchzuführen, und es fehlt an kurzen, wirksamen psychosozialen Interventionen, die in einer vollen Praxis sinnvoll durchführbar sind. Hier könnten in Zukunft digitale An- gebote die Face-to-face-Versorgung segensreich ergänzen.

3. Nicht für alle pädiatrischen Schmerzerkrankungen sind Leitlinien publiziert, die evidenzbasierte Diagnoseund Therapiepfade enthalten. Die S3-Leitlinie „Rückenschmerz bei Kindern und Jugendlichen" (https:// www.awmf.org/leitlinien/detail/ anmeldung/1/1l/027-070.html), die S2k-Leitlinie „Muskuloskelettale Schmerzen bei Kindern und Jugendlichen" (https://www.awmf.org/ leitlinien/detail/1l/027-073.html) und die S1-Leitlinie „Bauchschmerzen bei Kindern" (https://www.awmf. org/leitlinien/detail/ll/064-016.html) sind hier nur ein Anfang. Weitere Leitlinien müssen folgen. Ihr Praxistransfer muss wissenschaftlich begleitet werden.

4. Je stärker die Chronifizierung zunimmt, desto mehr sind psychosoziale Interventionen sowie ein interdisziplinäres Vorgehen gefragt. Die flächendeckende Versorgung mit niedergelassenen Kinder- und Jugendlichenpsychotherapeut ${ }^{*}$ innen muss unbedingt weiterverfolgt werden. Teilerfolge sind hier schon erzielt worden. Zeitgleich sind Angebote der Zusatzqualifikation zur speziellen Schmerzpsychotherapeut ${ }^{*}$ in notwendig, wie sie von der Deutschen Gesellschaft für psychologische Schmerztherapie und -forschung (DGPSF) auch gezielt für Kinder- und Jugendlichenpsychotherapeut ${ }^{\star}$ innen angeboten werden 
(https://www.dgpsf.de/fort-undweiterbildung).

5. Für invalidisierende chronische Schmerzen im Kindes- und Jugendalter ist die multimodale interdisziplinäre Schmerztherapie der Goldstandard [4]. Mit dem Deutschen Kinderschmerzentrum in Datteln (https://www. deutsches-kinderschmerzzentrum. de/), dem Kinderschmerzzentrum Baden-Württemberg in Stuttgart (https://www.klinikumstuttgart.de/kliniken-institutezentren/paediatrie-1-paediatrischeneurologie-psychosomatik-undschmerztherapie/kinderschmerz zentrum-baden-wuerttemberg), dem Bayrischen Kinderschmerzzentrum in Augsburg (https://www.ukaugsburg.de/zentren/bayerischeskinderschmerzzentrum/ueberblick. html) sowie dem Zentrum für Schmerztherapie junger Menschen in Garmisch (https://www.rheumakinderklinik.de/schmerz-zentrum) stehen überregional arbeitende pädiatrische Schmerzzentren zur Verfügung - weitere Zentren müssen folgen. Erfreulicherweise ist die „OPS 8.918 Interdisziplinäre multimodale Schmerztherapie " in diesem Jahr auf Betreiben von GKind (https:// www.gkind.de/) der Wirklichkeit der Pädiatrie weiter angepasst worden (https://www.icd-code.de/ops/code/ 8-918.html). Erneute Anpassungen, die den Blick weiten auf das Familiensystem, müssen folgen.

6. Viele Patient ${ }^{\star}$ innen mit invalidisierenden chronischen Schmerzen weisen starke psychiatrische Komorbiditäten wie Essstörungen, dissoziative Störungen oder schwere Depressionen auf. Die „offenen“ psychosomatischen Angebote der bestehenden pädiatrischen Schmerzzentren sind für diese Patienten ungeeignet. Es bedarf dringend kooperativ arbeitender kinder- und jugendpsychiatrischer Einheiten, die auch eine hohe Expertise zu chronischen Schmerzen aufweisen.

7. Chronische Schmerzen bei Kindern und Jugendlichen sind nicht der kleine Bruder oder die kleine
Schwester von chronischen Schmerzen bei Erwachsenen. Praktische und wissenschaftliche Erkenntnisse aus der Versorgung und Forschung mit erwachsenen Schmerzpatienten dürfen nicht kritiklos auf Kinder und Jugendliche übertragen werden. So scheint beispielsweise der Nutzen einer medikamentösen Migräneprophylaxe bei Erwachsenen bewiesen, bei Kindern und Jugendlichen widerspricht ein solches Standardvorgehen der gültigen Evidenzlage [2]. Es bedarf also einer fokussiert pädiatrischen Schmerzforschung, um nicht wegen fehlender pädiatrischer Daten in die missliche Lage zu kommen, Evidenz aus dem Erwachsenenbereich auf Kinder zu übertragen. Die pädiatrische Schmerzforschung hat die Chance, die Besonderheiten der Kindheit und Jugend zu fokussieren: a. Medikamente wirken im Kindesalter oft anders oder weisen eine andere Pharmakokinetik bzw. andere Nebenwirkungen auf als bei Erwachsenen,

b. Eltern und die Peer-Gruppe spielen für Kinder und Jugendliche eine außergewöhnliche Rolle,

c. Kinder und Jugendliche sind medienaffiner und -geübter,

d. ihre neuronale Plastizität erlaubt schnelle richtungsweisende Veränderungen hin zum Positiven,

e. Spaß und Witz sind wichtig für Compliance und Therapieerfolg und

f. das Erlernen von positivem Coping und das Steigern der Resilienz könnten Kinder stark machen für ihr ganzes Leben bis ins Erwachsenenalter.

Glücklicherweise fördert der Innovationsfonds Projekte, die diese Besonderheiten adressieren. Beispiele sind:

- mona - Entwicklungsbezogene, multimodale, interdisziplinäre Frühintervention im Rahmen eines Strukturierten Therapiekonzeptes für Kinder mit Migräne (https://innovationsfonds.g-ba.de/ projekte/neue-versorgungsformen/ moma-entwicklungsbezogenemultimodale-interdisziplinaere- fruehintervention-im-rahmeneines-strukturierten-therapiekonzep tes-fuer-kinder-mit-migraene-modules-on-migraine-activity.189)

- SCHMERZ-NETZ - Kinderschmerztherapie vernetzt: Sozialmedizinische Nachsorge für schwer chronifizierte pädiatrische Schmerzpatienten (https:// innovationsfonds.g-ba.de/projekte/ neue-versorgungsformen/schmerznetz-kinderschmerztherapievernetzt-sozialmedizinischenachsorge-fuer-schwer-chronifiziertepaediatrische-schmerzpatienten.185)

- Wissen(s)Star - Wissen macht stark! Empowerment von Eltern und Kind bei funktionellen Bauchschmerzen (https://innovationsfonds.g-ba.de/ projekte/versorgungsforschung/ wissen-s-star-wissen-machtstark-empowerment-von-elternund-kind-bei-funktionellenbauchschmerzen.315) oder

- Feed-bApp - Einfluss unterschiedlich (kosten)-intensiver FeedbackFunktionen einer pädiatrischen Schmerz-App auf die Versorgungsqualität (https://innovationsfonds.gba.de/downloads/media/231/Listegefoerderter-Projekte_VSF_FBK2019-12-12.pdf)

Es ist zu erwarten, dass sich aus den großen vom Innovationsfonds geförderten Projekten sowie Forschungsanstrengungen, die von der Deutschen Forschungsgemeinschaft, dem Bundesministerium für Bildung und Forschung und Stiftungen gefördert werden, maßgebliche positive Veränderungen der Versorgung ergeben. Kindern und Jugendlichen mit chronischen Schmerzen sei es zu wünschen.

\section{Korrespondenzadresse}

Prof. Dr. B. Zernikow

Lehrstuhl für Kinderschmerztherapie und Pädiatrische Palliativmedizin, Universität Witten/Herdecke

Witten, Deutschland

B.Zernikow@kinderklinik-datteln.de

Interessenkonflikt. B. Zernikow gibt an, dass kein Interessenkonflikt besteht. 


\section{Literatur}

1. Höfel L, Draheim N, Haas JP, Ebinger F (2020) Schmerzmedizinische Versorgung chronisch schmerzkranker Kinder und Jugendlicher in Deutschland: Eine Bestandsaufnahme. Schmerz. https://doi.org/10.1007/s00482-020-00510-9

2. LocherC, KossowskyJ, Koechlin H, Lam TL, BarthelJ, Berde CB, Gaab J, Schwarzer G, Linde K, Meissner K (2020) Efficacy, safety, and acceptability of pharmacologic treatments for pediatric migraine prophylaxis: a systematic review and network meta-analysis. JAMA Pediatr 174(4):341-349. https:// doi.org/10.1001/jamapediatrics.2019.5856

3. Selent F, Schenk S, Genent D, Wager J, Zernikow B (2020) Diagnostik und Therapie bei Kindern und Jugendlichen mit chronischem Schmerz: Zeitliche Entwicklung potenziell gesundheitsgefährdender Maßnahmen. Schmerz. https://doi.org/10.1007/ s00482-020-00506-5

4. Stahlschmidt L, Zernikow B, Wager J (2016) Specialized rehabilitation programs for children and adolescents with severe disabling chronic pain: indications, treatment and outcomes. Children 3(4):33. https://doi.org/10.3390/children3040033

Aktuelle Buchempfehlungen zur Schmerzmedizin aus dem SpringerVerlag

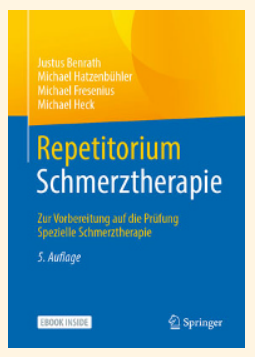

\section{Repetitorium Schmerztherapie}

Benrath, J., Hatzenbühler, M., Fresenius, M., Heck, M. | 5. Aufl. 2020, XIV, 272 S., 38 Abb., Softcover 44,99€, ISBN 978-3-662-61782-3

Das Buch vermittelt in knapper und verständlicher Form die Grundlagen und Besonderheiten der speziellen Schmerztherapie. Die Inhalte orientieren sich an den Weiterbildungsinhalten der BÄK für die ZusatzWeiterbildung "Spezielle Schmerztherapie“. Das Buch eignet sich hervorragend zur Prüfungsvorbereitung, ist aber auch ein wertvoller Begleiter im Klinikalltag aller schmerztherapeutisch tätigen Ärzte.

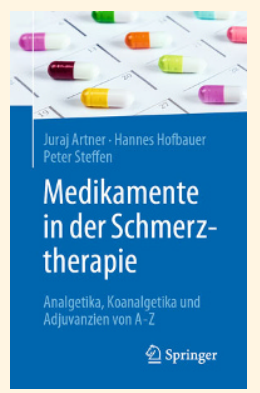

\section{Medikamente in der Schmerztherapie}

Artner, Juraj, Hofbauer, Hannes, Steffen, Peter| 1. Aufl. 2020, XXIV, 460 S. 3 Abb., Softcover 54,99€, ISBN 978-3-662-61691-8

Das Werk beschreibt 100 in der Schmerztherapie eingesetzte Pharmaka nach ihrem Wirkprofil. Der Focus liegt auf den für die Praxis relevanten Fakten und auch Off-Label-Anwendungen der Medikamente werden beleuchtet. Darüber hinaus liefert das Werk Tipps für den richtigen Einsatz, Entscheidungshilfen für die richtige Medikamentenwahl sowie Informationen bei besonderen Fragestellungen.

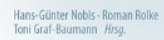

Schmerz-eine Herausforderung
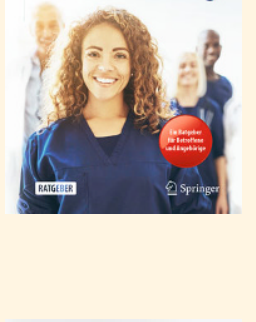

Erfolgreich gegen Kopfschmerzen und Migräne -
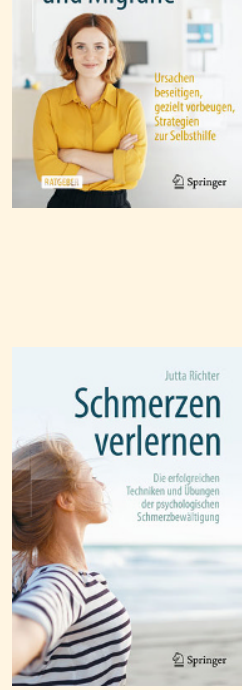

\section{Schmerz - eine Herausforderung}

Nobis, Hans-Günter, Rolke, Roman, Graf-Baumann, Toni (Hrsg.) | 3. Aufl. 2020, XVII, 204 S., 52 Abb., Softcover 19,99€, ISBN 978-3-662-60400-7 In diesem Ratgeber erfahren Menschen mit chronischen Schmerzen und deren Angehörige mehr über die körperlichen, psychischen und sozialen Zusammenhänge von Schmerz. Das Werk ist von der Deutschen Schmerzgesellschaft e.V., der Deutschen Gesellschaft für Psychologische Schmerztherapie und -forschung sowie der Deutschen Migräne- und Kopfschmerzgesellschaft empfohlen.

\section{Erfolgreich gegen Kopfschmerzen und Migräne}

Göbel, Hartmut | 9. Aufl. 2020, XIII, 558 S., 176 Abb., Softcover 29,99€, ISBN 978-3-662-61687-1

Das Buch informiert über Formen und Ursachen von Kopfschmerzen und Migräne sowie über moderne Diagnosemöglichkeiten und Therapieverfahren, inklusive alternativer Behandlungsverfahren. Darüber hinaus erhalten Betroffene wichtige Informationen und Tipps zur Selbsthilfe und Vorbeugung. Dazu gehören u.a. zahlreiche Adressen von Selbsthilfegruppen und Kliniken, Tipps zur richtigen Einnahme von Medikamenten und Hinweise auf wertvolle Serviceseiten im Internet.

\section{Schmerzen verlernen}

Richter, Jutta | 4. Aufl. 2021, X, 144 S. 94 Abb., Softcover 19,99€, ISBN 978-3-662-62686-3

Dieses Buch zeigt konkrete Techniken und Übungen der psychologischen Schmerzbewältigung. Es wendet sich an alle Schmerzpatienten, die Schmerzen aktiv bewältigen wollen und an alle Therapeuten und Ärzte, die ihre Patienten dabei unterstützen möchten. Dargestellt werden über 30 Techniken und Übungen, wie Achtsamkeitsübungen, Entspannungstechniken oder Hypnoseverfahren. 